\title{
Eosinophilic Endomyocarditis: A Rare Case of Neonatal Mortality
}

\author{
Allison J. Pollock, MD ${ }^{1}$ Stacy L. Hitt, MD² Michael A. Stier, MD ${ }^{3} \quad$ Laura M. Houser, MD ${ }^{1}$ \\ ${ }^{1}$ Department of Pediatrics, University of Wisconsin School of Medicine \\ and Public Health, Madison, Wisconsin \\ 2 Department of Pathology, Children's Hospital of Wisconsin, \\ Milwaukee, Wisconsin \\ ${ }^{3}$ Department of Pathology, University of Wisconsin School of \\ Medicine and Public Health, Madison, Wisconsin

\begin{abstract}
Address for correspondence Allison J. Pollock, MD, Division of Pediatric Endocrinology, Department of Pediatrics, University of Wisconsin School of Medicine and Public Health, 600 Highland Avenue, Box 4108, Madison, WI 53792 (e-mail: apollock@pediatrics.wisc.edu).
\end{abstract} \\ Am J Perinatol Rep 2015;5:e150-e152.
}

\begin{abstract}
Background Eosinophilic endomyocarditis (EEM) is a rare diagnosis that is extremely uncommon in newborns. This case report aimed to present a case of neonatal mortality from acute cardiac failure due to EEM.

Case Our report presents a term male neonate with minor complications in the immediate postnatal course, who was discharged at 48 hours of life, but who developed unexpected respiratory distress, followed by cardiac arrest and death at 3 days of life. One day after discharge, the infant developed respiratory distress and cool skin, and then developed cardiac arrest at the pediatrician's office, undergoing resuscitation with intravenous fluid, cardiopulmonary resuscitation, epinephrine, atropine, and failed intubation. Autopsy revealed EEM, an inflammatory infiltrative process involving the

\section{Keywords}

- eosinophilic endomyocarditis

- myocarditis

- erythema toxicum

- cardiac arrest

- hypereosinophilic syndrome endomyocardium.

Pathology Pathogenesis involves three stages: (1) myocarditis with an acute eosinophilic inflammatory infiltrate followed by (2) myocyte necrosis and eventually (3) fibrosis in the final stage of the disease.

Discussion The cause of death was acute cardiac failure due to intense eosinophilic infiltration and degranulation with early subendocardial myocyte necrosis but before development of extensive myocyte necrosis. This case appears to be the youngest patient reported with EEM.
\end{abstract}

Eosinophilic endomyocarditis (EEM) is histologically diagnosed by eosinophilic infiltration of the endocardium and subendocardial myocardium. It is rare but can be seen in all ages ${ }^{1}$; infant cases are extremely rare. ${ }^{2}$ EEM is frequently associated with hypereosinophilic syndromes, such as leukemia, allergic disorders, and Loeffler endocarditis, and can present with peripheral eosinophilia., There is one published neonatal case of EEM: a 26-day-old infant presented with poor feeding and cardiopulmonary arrest without other organ involvement on postmortem exam. ${ }^{2}$

received

October 29, 2014

accepted after revision

March 26, 2015

published online

May 22, 2015
$10.1055 / \mathrm{s}-0035-1552929$ ISSN 2157-7005.

\section{Case}

Baby B was born to a 30-year-old married primigravida mother with normal routine prenatal laboratories. Mother reported asthma, allergies, anxiety, constipation, esophageal stricture, and gastroesophageal reflux. Family history was negative for heart disease. Pregnancy was complicated by fetal bradycardia, heart rates under $120 \mathrm{bpm}$ at $33^{4 / 7}$ weeks, but with normal fetal movement and unremarkable antenatal ultrasounds, including four-chamber heart view at 20 weeks' gestation. Due to low baseline fetal heart rate, serial fetal

Copyright $\odot 2015$ by Thieme Medical Publishers, Inc., 333 Seventh Avenue, New York, NY 10001, USA. Tel: +1(212) 584-4662.
License terms

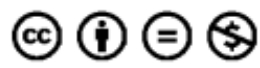


growth studies, nonstress tests, and biophysical profiles were performed. These were found to be reassuring.

Presentation to obstetric triage at $39^{1 / 7}$ weeks' gestation was due to recent history of vomiting and diarrhea, since resolved. However, induction of labor was performed for nonreactive nonstress test and fetal heart rate of 105. Oxytocin administration and artificial rupture of membranes occurred at 6 hours before delivery with no fluid return, but with meconium present and one loose nuchal cord at delivery. APGAR scores were 9 and 9 and heart rate was greater than 100. Birth weight was 2,892 g (15.9th World Health Organization [WHO] percentile), length was $49.5 \mathrm{~cm}$ (42.1st WHO percentile), and head circumference was $34 \mathrm{~cm}$ (34.5th WHO percentile). The placenta was visually normal with threevessel umbilical cord.

In the postnatal course, mild jaundice developed by 8 hours of age, with normal voiding, stooling, and vigor at breastfeeding. A rash with erythematous macules and patches with central yellow papules was clinically diagnosed as erythema toxicum. Recorded heart rate was between 108 and 168 per minute and respiratory rate between 38 and 62 per minute. Remainder of vital signs and exam findings were normal. The filter paper newborn screen test later resulted as normal. Congenital heart disease screening initially showed low preductal pulse oximetry at $94 \%$ at 25 hours but was normal (100\%) at 35 hours of age. Total bilirubin was $9.0 \mathrm{mg} /$ $\mathrm{dL}$ at 36 hours of age, below phototherapy treatment guidelines. The infant failed bilaterally on hearing screening and outpatient exam was scheduled. The rash was fading by discharge, at approximately 41 hours of age, weighing $2,806 \mathrm{~g}$ (3\% weight loss since birth) in good condition. Follow-up bilirubin level and weight check were planned for 1 to 2 days after discharge.

One day after discharge, at a local clinic, mother reported concern for cold skin. Respiratory distress with stridor of unknown duration was appreciated with respiratory failure. The pediatrician began mouth-to-mouth resuscitation. Emergency Medical Service was called. The infant developed pulseless cardiac arrest and underwent unsuccessful cardiopulmonary resuscitation including failed intubation, compressions, medication administration, and fluid resuscitation. Although intubation was attempted multiple times, this was unsuccessful due to swollen epiglottis. Bag-mask ventilation continued, with poor air exchange appreciated. One hour after presentation, the infant was pronounced dead.

\section{Pathology}

Postmortem examination demonstrated a normally developed male with anatomic parameters appropriate for gestational age, without rash. External exam findings were consistent with resuscitation. Internal examination showed a moderate amount of straw-colored pleural, pericardial, and peritoneal fluid. Epiglottic swelling was not confirmed, nor was mucus plugging of the airways present. Multifocal gastrointestinal hemorrhage and acute bilateral renal papillary necrosis were identified interpreted as manifestations of cardiac failure. Postmortem comprehensive toxicologic screening of venous blood was negative. Nasopharyngeal and colorectal swabs were negative for adenovirus, cytomegalovirus, enteroviruses, herpes simplex virus, and varicellazoster virus. Nasopharyngeal swabs were also negative for influenza A and B; parainfluenza 1,2,3; and respiratory syncytial virus. The heart and lungs weighed $90.25 \mathrm{~g}$ (expected weight $85.25 \mathrm{~g}$ ). Microscopic heart sections were notable for an eosinophilic inflammatory infiltrate involving the endocardial aspect of the myocardium, focally associated with acute extravasation of blood and early subendocardial myocyte necrosis (-Fig. 1). Microscopic studies were otherwise normal. No vascular inflammation, thrombosis, microorganisms, or viral inclusions were present. The lungs revealed diffuse congestion and perimortem hemorrhages. The liver and spleen demonstrated mildly increased eosinophils; however, no intravascular eosinophilic infiltrates were found in any other tissues examined.

\section{Discussion}

This healthy-appearing male infant developed symptomatic heart failure and cardiopulmonary arrest within 24 hours of hospital discharge. His rapid deterioration and postmortem diagnosis of EEM prompted review of his case for clues to explain his unexpected unfortunate outcome and illuminate warning signs. The prenatal history and persistent low heart rate with otherwise normal exam did not raise specific clinical concerns for heart failure after birth. The infant's erythema toxicum rash (known to be eosinophil mediated $)^{5,6}$ was more prominently distributed and arose earlier than seen routinely, but was otherwise consistent with this common benign newborn rash, and resolved by time of death. There were no other signs of extracardiac hypereosinophilia. Given what is known about EEM, there were no clear risk factors or warning signs, although his bradycardia, initial low oxygen saturations on congenital heart screen, early and prominent erythema toxicum rash, and failed hearing screen were notable. There are case reports of eosinophilic granulomas forming in the middle

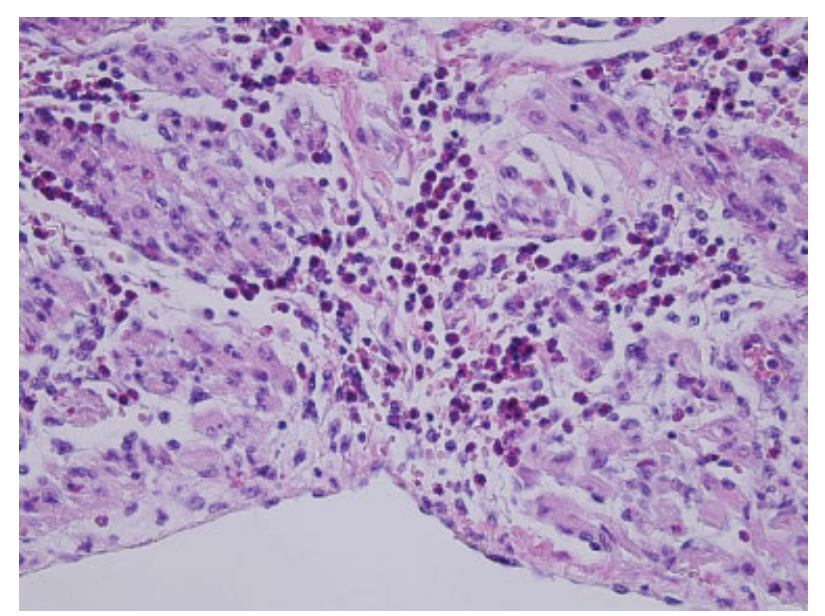

Fig. 1 Endomyocardial surface of the heart showing eosinophil infiltration, magnified $\times 400$. 
ear of patients with idiopathic hypereosinophilic syndromes, which is one plausible explanation of the failed hearing screen. ${ }^{7,8}$ In addition, mother's history of esophageal stricture and reflux are interesting to note, as these clinical findings can often overlap with findings of eosinophilic esophagitis. The infant's mother has not been evaluated for hypereosinophilic syndromes and there has not been any testing for the $4 \mathrm{q} 12$ deletion, a known genetic cause of hypereosinophilic syndromes.

The pathogenesis of EEM involves three stages beginning with (1) an intense myocarditis and acute eosinophilic inflammatory infiltrate followed by (2) myocyte necrosis developing approximately 5.5 weeks after onset of symptom, and eventually (3) fibrosis in the final stage of the disease. ${ }^{9}$ The "intense" myocarditis during the first stage refers to the presence of inflammatory cells (eosinophils) in the patient's cardiac histology. The necrotic second stage of the disease is associated with high mortality due to cardiac deterioration. Over several months, thrombotic material may form at the damaged endocardium as the inflammation abates. Cardiac remodeling and fibrosis occur in the third and final stages, which may progress to a dilated and even a restrictive cardiomyopathy in cases with extensive endomyocardial fibrosis. Although typically associated with hypereosinophilia, rare cases of EEM have been reported without it. $^{2,3,10}$ Loeffler syndrome, one variation of restrictive EEM, could present similarly to this infant; however, the infant was still in the acute stage and had not yet developed fibrosis or restrictive physiology associated with Löeffler syndrome. Based on the histologic findings and natural history of the disease, this infant appears to have experienced acute cardiac failure in the early stage of the disease process due to the intensity of the eosinophilic infiltration and degranulation with early subendocardial myocyte necrosis, before extensive myocyte necrosis could take place.

This case appears to be the youngest patient ever reported with EEM. Lie and Hunt report an adult case of eosinophilic endomyocarditis which was associated with conduction abnormalities; this patient died of myocardial infarction and cardiogenic shock. Unexpectedly on autopsy, EEM was discovered. ${ }^{11}$ The only other neonatal case of EEM in the literature is a 26-day-old infant with a brief history of poor feeding leading to cardiorespiratory arrest. The finding of only an eosinophilic infiltrate in the myocardium on postmortem makes it similar to our case. ${ }^{2}$ Perhaps the fetal bradycardia and nonreactivity were subtle clues pointing toward poor cardiovascular adaptation to EEM, but given the paucity of neonatal cases, and unclear natural history of neonatal EEM, the jury is still out.

\section{Financial Support}

The authors have no conflicts of interest or financial relationships to declare.

\section{Acknowledgments}

The authors thank Dr. Frank Greer, Dr. Pam Kling, Dr. Don Singer, and Dr. Shardha Srinivasan for their valuable expertise and guidance.

\section{References}

1 Henri C, Roméo P, Chin A, Dore A, Ducharme A. Eosinophilic endomyocarditis: an unusual cause of heart failure in a young patient. Can J Cardiol 2011;27(3):390.e15-390.e16

2 Krous HF, Haas E, Chadwick AE, Wagner GN. Sudden death in a neonate with idiopathic eosinophilic endomyocarditis. Pediatr Dev Pathol 2005;8(5):587-592

3 Priglinger U, Drach J, Ullrich R, Baumgartner H, Huber K, Maurer G. Idiopathic eosinophilic endomyocarditis in the absence of peripheral eosinophilia. Leuk Lymphoma 2002;43(1):215-218

4 Spiegel R, Miron D, Fink D, Gavriel H, Horovitz Y. Eosinophilic pericarditis: a rare complication of idiopathic hypereosinophilic syndrome in a child. Pediatr Cardiol 2004;25(6): 690-692

5 Marchini G, Nelson A, Edner J, Lonne-Rahm S, Stavréus-Evers A, Hultenby K. Erythema toxicum neonatorum is an innate immune response to commensal microbes penetrated into the skin of the newborn infant. Pediatr Res 2005;58(3):613-616

6 Nelson A, Ulfgren AK, Edner J, et al. Urticaria neonatorum: accumulation of tryptase-expressing mast cells in the skin lesions of newborns with erythema toxicum. Pediatr Allergy Immunol 2007;18(8):652-658

7 Kimitsuki T, Kakazu Y, Komune S. Idiopathic hypereosinophilic syndrome involving the middle ear: clinical case report and discussion. Acta Otolaryngol 2001;121(4):539-542

8 Takayama K, Yadohisa O, Furuno T, et al. Case report: the first report of idiopathic hypereosinophilic syndrome involved with lung and middle ear. Am J Med Sci 1995;309(5):282-284

9 Silver M, Gotlieb AI, Schoen FR. Cardiovascular Pathology. 3rd ed. London, England: Churchill Livingstone Elsevier; 2001

10 deMello DE, Liapis H, Jureidini S, Nouri S, Kephart GM, Gleich GJ. Cardiac localization of eosinophil-granule major basic protein in acute necrotizing myocarditis. N Engl J Med 1990;323(22): $1542-1545$

11 Lie JT, Hunt D. Eosinophilic endomyocarditis complicating acute myocardial infarction. Involvement of the cardiac conduction system. Arch Intern Med 1974;134(4):754-757 